\title{
Observation of well-defined quasiparticles at a wide energy range in a quasi-two-dimensional system
}

\author{
L.-L. Jia, ${ }^{1}$ Z.-H. Liu, ${ }^{1}$ Y.-P. Cai, ${ }^{1}$ T. Qian, ${ }^{2}$ X.-P. Wang, ${ }^{2}$ H. Miao, ${ }^{2}$ P. Richard,${ }^{2,3}$ Y.-G. Zhao, ${ }^{1}$ Y. Li, ${ }^{1}$ D.-M. Wang, ${ }^{1}$ J.-B. He, ${ }^{1}$ \\ M. Shi, ${ }^{4}$ G.-F. Chen, ${ }^{1,2,3}$ H. Ding, ${ }^{2,3}$ and S.-C. Wang ${ }^{1,5}$ \\ ${ }^{1}$ Department of Physics, Renmin University of China, Beijing 100872, China \\ ${ }^{2}$ Beijing National Laboratory for Condensed Matter Physics, and Institute of Physics, Chinese Academy of Sciences, Beijing 100190, China \\ ${ }^{3}$ Collaborative Innovation Center of Quantum Matter, Beijing, China \\ ${ }^{4}$ Swiss Light Source, Paul Scherrer Institute, CH-5232 Villigen, Switzerland \\ ${ }^{5}$ Beijing Key Laboratory of Opto-electronic Functional Materials and Micro-nano Devices, Renmin University of China, Beijing, China
}

(Received 7 March 2014; revised manuscript received 24 June 2014; published 25 July 2014)

\begin{abstract}
We report an observation of a highly anisotropic Dirac-cone structure, and its unconventional electronic behaviors, in high quality $\mathrm{SrMnBi}_{2}$ crystal by angle-resolved photoemission spectroscopy measurement. We observed a well-defined sharp quasiparticle peak in a wide energy range, linearly dispersive, forming a highly anisotropic holelike Dirac cone $\left(v_{F_{1}} / v_{F_{2}} \sim 5-6\right)$. The density of state remains linear, and the scattering rate of the quasiparticle increases linearly as a function of binding energy up to higher binding energy. The results contrast with the expectation for a two-dimensional (2D) or three-dimensional (3D) Fermi-liquid theory and rather suggest the existence of a two-dimensional electron gas system in this 3D material, making $\mathrm{SrMnBi}_{2}$ a potentially useful 2D-electron-gas-encapsulated material.
\end{abstract}

DOI: 10.1103/PhysRevB.90.035133

PACS number(s): 71.20.-b, 71.18.+y, 73.20.At

The description of condensed matter systems in the manybody limit is simplified by using the concept of quasiparticles (QPs), which represent an elementary fermionic excitation behaving like a particle [1-3]. In a Fermi liquid (FL), these QPs have a finite lifetime decreasing quadratically with energy [4,5]. Despite recent enthusiasm generated by the discovery of a plethora of new materials exhibiting Dirac cones with exotic properties [6-9], how the concept of QPs can be extended to low-dimensional systems characterized by Dirac physics remains unclear.

Recently, Dirac-particle behavior with linear energy dispersion was predicted and observed in $\mathrm{SrMnBi}_{2}$ and $\mathrm{CaMnBi}_{2}$ $[10,11]$. Indeed, large magnetoresistance, high mobility, and small cyclotron resonant mass suggest the existence of Dirac fermions in these materials [12,13]. Unlike the nearly symmetric Dirac cones in graphene though, angle-resolved photoemission spectroscopy (ARPES) measurements in $\mathrm{SrMnBi}_{2}$ [11] reveal a strong anisotropy in the Dirac-cone dispersion, which local-density approximation (LDA) calculations attribute to the Bi $6 p$ orbitals in the SrBi layer $[11,12,14]$. Both ARPES and thermopower measurements have reported the existence of small Fermi surface (FS) with linear dispersion, but are conflicting on the sign of carriers [11,14]. Quantum oscillation experiments also suggest a small FS compatible with Dirac points in the vicinity of the Fermi energy $\left(E_{F}\right)[10]$.

In this paper, we report an observation of a two-dimensional (2D) electron gas (2DEG) on a bulk material. The detailed ARPES study on bulk material $\mathrm{SrMnBi}_{2}$ reveals a highly anisotropic Dirac fermion in the Bi square net. Dirac-cone-like linear dispersions were observed with highly anisotropic Fermi velocities $v_{F_{1}} / v_{F_{2}} \sim 5-6$. In a wide energy range ( $\sim 650 \mathrm{meV}$ ), we observed a well-defined quasiparticle peak with nearly energy-independent scattering rate (inverse of linewidth), and linear increasing density of states (DOS). The energy independent of the QP scattering rate is inconsistent with FL description. All of these observations suggest an unconventional non-FL 2DEG in the bulk system.
High quality single crystals of $\mathrm{SrMnBi}_{2}$ were grown by the flux method. X-ray diffraction data were collected with $\mathrm{Cu} K \alpha$ radiation at room temperature to confirm that the samples are from a single phase. Samples were cleaved in situ yielding a flat (001) surface. ARPES measurements were performed at Renmin University of China using a Scienta R3000 analyzer with a He discharging lamp (He-I $\alpha$ line, $h v=21.2 \mathrm{eV})$, at the PGM beamline of the Synchrotron Radiation Center (WI) using a Scienta R4000 analyzer, and at the SIS beamline at Swiss Light Source equipped with a R4000 analyzer. Samples were measured at low temperature with $T=30 \mathrm{~K}$ for FS mapping and $T=10 \mathrm{~K}$ for high-resolution measurement. The high-resolution ARPES measurements were conducted with energy resolution better than $5 \mathrm{meV}$ and angular resolution better than $0.2^{\circ}$, which corresponds to a momentum resolution of $\Delta k \leqslant 0.009 \AA^{-1}$ at photon energy $h v=30 \mathrm{eV}$.

The crystal structure of $\mathrm{SrMnBi}_{2}$, as shown in Fig. 1(a), consists of alternating $\mathrm{SrBi}$ and $\mathrm{MnBi}$ layers, with the unit cell doubling along the $c$ axis due to the opposite orientation of the $\mathrm{MnBi}_{4}$ tetrahedra. The $\mathrm{Bi}$ atoms in the $\mathrm{SrBi}$ layer, separated by a distance of $3.239 \AA$, form a square net believed to be responsible for the interesting electronic states near the Fermi energy [14]. Well-defined peaks in the photoemission core level spectrum are displayed in Fig. 1(b), which can be assigned easily in the 3-30 eV range of binding energy. We note that the clear asymmetry in the $\mathrm{Bi} 5 d$ peaks comes from the existence of two inequivalent $\mathrm{Bi}$ sites [shown as $\mathrm{Bil}$ and $\mathrm{Bi} 2$ in Fig. 1(a)] in the system. The more dispersive electronic states close to $E_{F}$ are mainly from the $\mathrm{Bi} 6 p_{1 / 2}$ and the $\mathrm{Mn}$ $3 d$ orbitals [14]. Figure 1(c) shows the integrated intensity within $\pm 20 \mathrm{meV}$ of $E_{F}$, which approximately represents the FS of the material, at low temperature $(T=30 \mathrm{~K})$ and at photon energy $h v=60 \mathrm{eV}$ ( $\Gamma$ plane), similar FS topology is achieved at $h v=30 \mathrm{eV}$. As we can see later from Fig. 3(a), the value of $k_{F}$ of the Dirac-like dispersion remains the same as that in Figs. 1(c) and 2(a), suggesting little $k_{z}$ dependence in the $\mathrm{SrMnBi}_{2}$ system. From Fig. 1(c), two kinds of FSs 
(a)

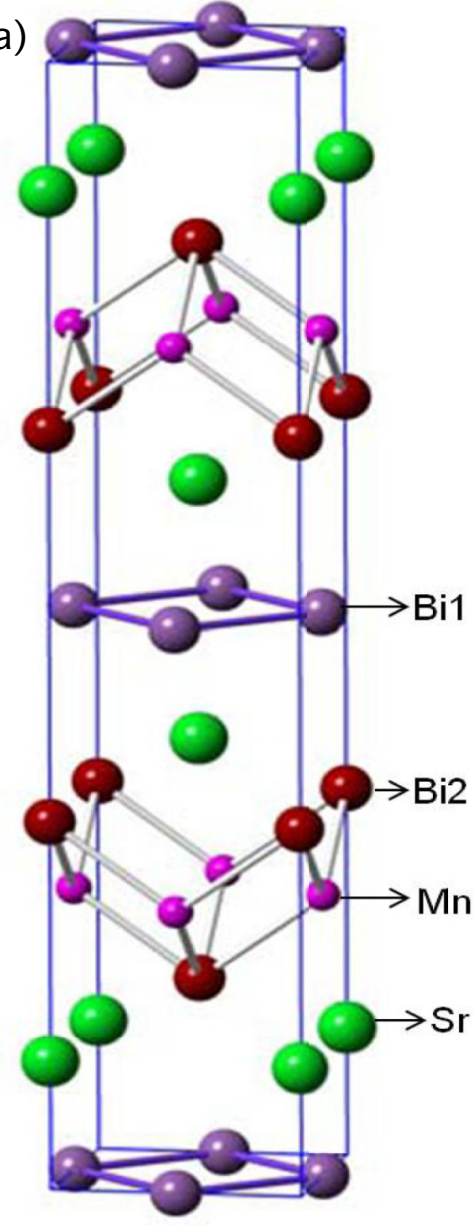

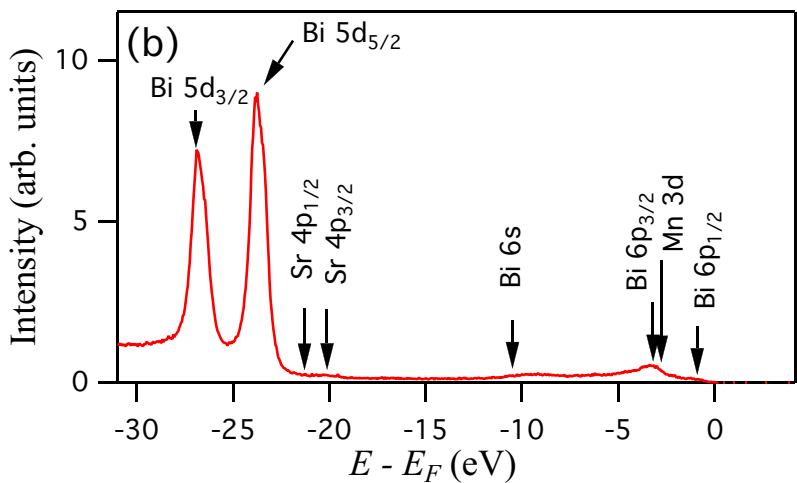

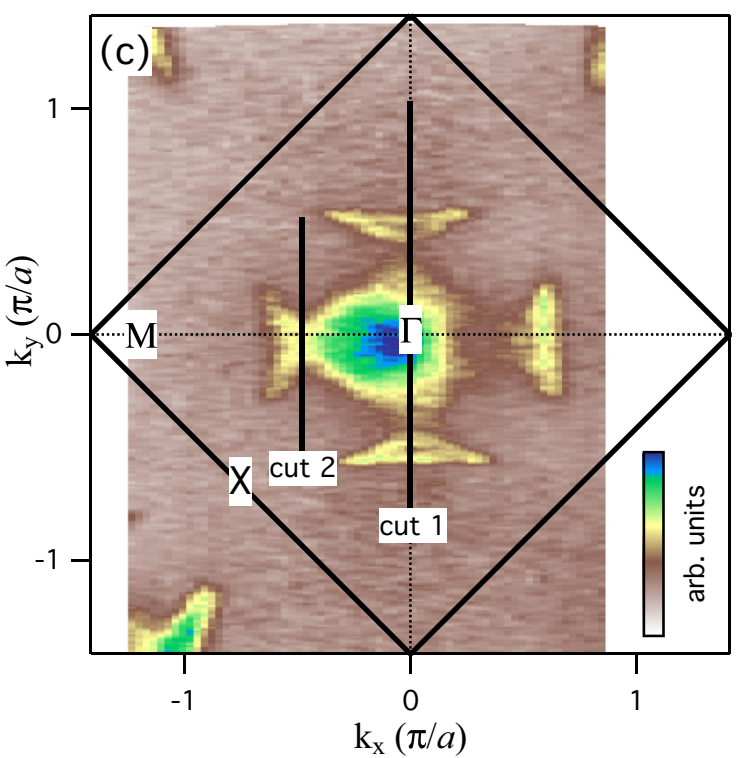

FIG. 1. (Color online) Structure, core levels, and Fermi surface topology of $\mathrm{SrMnBi}_{2}$ at low temperature $(T=30 \mathrm{~K})$. (a) Crystal structure of $\mathrm{SrMnBi}_{2}$ with $\mathrm{MnBi}_{4}$ layers and Bi square nets. (b) Shallow core levels of the $\mathrm{SrMnBi}_{2}$. (c) Integrated intensity plot around $E_{F}(-20$ meV, $20 \mathrm{meV}$ ), which approximately illustrates the FS topology of $\mathrm{SrMnBi}_{2}$, with photon energy $h v=60 \mathrm{eV}$. The primary BZ is illustrated by black lines in (c).

can be distinguished: a large diamond-shaped FS centered at the Brillouin zone (BZ) center $(\Gamma)$ originating from two bands mainly attributed to the Mn $3 d$ orbitals [14], whose band dispersion will be discussed later in detail, and four small crescentlike FSs located along $\Gamma-M[(0,0)-(\pi, \pi)]$ and equivalent directions, with their long sides perpendicular to $\Gamma-M$. The four small FSs occupy $6.3 \%$ of the BZ area. The observed FS topology is consistent with quantum oscillation measurements and LDA calculations in the antiferromagnetic configuration $[12,15]$.

We display in Fig. 2 the electronic band dispersion along high symmetry lines at low temperature $(T=30 \mathrm{~K})$ with photon energy $h v=60 \mathrm{eV}$. The ARPES intensity plot, the corresponding second momentum derivative, and the momentum distribution curves (MDCs) along cut 1, which is along $\Gamma-M$ and perpendicular to the crescent FS, are shown in Figs. 2(a)-2(c), respectively, to illustrate the band dispersion. We can identify two bands crossing $E_{F}$ near the BZ center: one electronlike band with minimum at $\Gamma$ and one holelike band, which are marked by dashed lines in Fig. 2(a). These two bands are mainly from the $\mathrm{Mn} 3 d$ orbitals located at the MnBi layer, forming the diamondlike FSs shown in Fig. 1(c). The two bands are nearly coincident at $E_{F}$, and the corresponding FSs are hardly distinguished from the intensity plot in Fig. 1(c).

Slightly away from the zone center, two linear bands dispersing in opposite directions converge near $E_{F}$ at $k_{\|}=$ $(0.37,0.37) \pi / a$. More precisely, linear extrapolation indicates that the apex of this Dirac-cone electronic dispersion locates $10 \mathrm{meV}$ above $E_{F}$. The inner band has lower intensity and is detected from $E_{F}$ to $E_{B} \sim 100 \mathrm{meV}$. The outer band has higher intensity and a linear dispersion up to about $650 \mathrm{meV}$ of binding energy with a well-defined sharp peak. At binding energy higher than $650 \mathrm{meV}$, the dispersion starts deviating from a linear dispersion and the peak becomes broader due to the proximity of other bands, as shown in Fig. 3(a).

Figures 2(d)-2(f) show, respectively, the intensity plot, the second derivative of intensity, and the MDCs along cut 2, the long direction of the crescent. Similarly to cut 1 , two dispersion branches with sharp peaks form a Dirac cone with apex slightly above $E_{F}$. However, the Fermi velocity $v_{F}$ is much smaller along that direction. While we find $v_{F}=$ $8.64 \mathrm{eV} \AA\left(1.3 \times 10^{6} \mathrm{~m} / \mathrm{s}\right)$ along cut 1 , which corresponds 

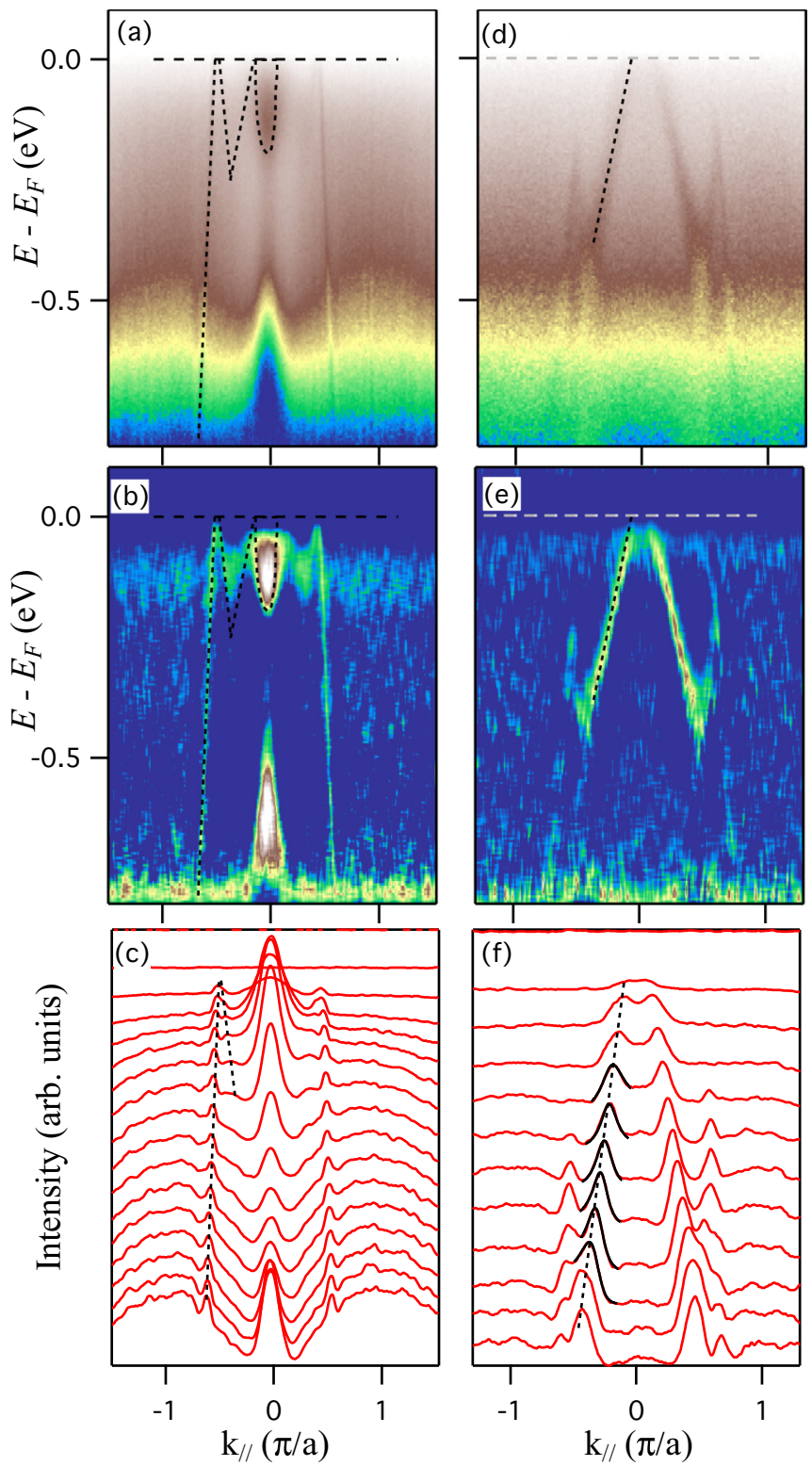

FIG. 2. (Color online) Band dispersion of anisotropic Dirac cone along two directions. (a)-(c) Intensity plot, second derivative $\left(\partial^{2} I / \partial \omega^{2}\right)$, and the MDCs along cut 1 , shown in Fig. 1(c), respectively. (d)-(f) The intensity plot, second derivative, and the MDCs plot along cut 2 . The dashed lines in figures are peak positions to illustrate band dispersions.

to $\sim 1 / 230$ of the speed of light, similar to that of graphene, we determine a velocity about five times slower along cut 2 $\left(V_{F}=1.78 \mathrm{eV} \AA \approx 1 / 1100 c\right)$. The Dirac cone is thus highly anisotropic. Although the existence of an anisotropic Dirac cone in $\mathrm{SrMnBi}_{2}$ has been previously reported [11], the FS topology and carrier type are different between our results and that previous report [11].

In order to study in more detail the sharp dispersive feature associated with the Dirac cone, we recorded additional data along cut 1 , using higher energy and momentum resolutions. Figure 3(a) shows the intensity plot at low temperature $(T=$ $10 \mathrm{~K}$ ) and photon energy $h v=30 \mathrm{eV}$. Its second momentum derivative intensity plot, displayed in Fig. 3(d), enhances the bulk bands. From the figures, a linear dispersion is observed over a wide range of energy, practically until the band reaches the bulk bands at high energy $(\geqslant 650 \mathrm{meV})$. To characterize the intrinsic properties of this dispersive feature, we removed a constant energy distribution curve (EDC) recorded away from the dispersive band assumed as a momentum-independent background. The result, illustrated in Fig. 3(b) for the linear dispersion part, shows a quite flat background with nearly zero intensity away from the band, as also illustrated by the corresponding background-removed EDCs shown in Fig. 3(e), thus justifying our approach.

We note that the peak splits by up to $0.016 \AA^{-1}$ close to $E_{F}$, which was not predicted by LDA calculations $[11,14]$. The size of the split decreases with an increase of binding energy and becomes indistinguishable at $E_{B} \sim 150 \mathrm{meV}$. This split can be seen in the MDC plots shown in Fig. 4(a) and the corresponding MDC fittings. Although the origin of this splitting remains unclear, we conjecture that it may be caused by bilayer interactions due to the doubling of the unit cell along the $c$ axis, or the Rashba splitting observed in the 2DEG system [16]. We roughly estimate the Rashba energy $E_{R}$ at about $150-200 \mathrm{meV}$ where the splitting becomes indistinguishable, which is indicated by the blue curves in Figs. 4(a) and 4(b). The $E_{R}$ has similar value as observed in other $\mathrm{Bi}$ on a $\mathrm{Ag}(111)$ surface $[16,17]$.

One of our most interesting observations is the nearly energy-independent QP spectral line shape down to about $650 \mathrm{meV}$ of binding energy. Indeed, Fig. 3(c) indicates that the momentum-integrated spectral intensity $n_{\theta}(\omega) \equiv$ $\int_{k=k(\theta)} n(k, \omega) d k$ remains almost constant over a wide energy range, which is consistent with a $2 \mathrm{D}$ electron system. The total density of states in a 2D system with linear dispersion $\omega=\omega_{0}+v_{0}\left(k-k_{F}\right)$ is linear as a function of binding energy

$$
\rho(\omega)=\frac{2 \pi}{v_{0}^{2}}\left(\omega-\omega_{0}\right) .
$$

Noticing that the size of the enclosed constant energy contour increases linearly with binding energy for a Dirac-cone-like dispersion, we thus estimate that the total density of states of the band increases linearly as a function of binding energy, as predicted by Eq. (1), and is not consistent with either 2D/3D Fermi liquid or 3D Dirac-like behavior as listed in Table I.

The most striking observation is the QP line shape. As shown in Fig. 3(e), where we show EDCs with a constant background removed, the QP line shape remains nearly identical in the energy range. Even at $E_{B} \sim 650 \mathrm{meV}$, the QP line shape is well maintained with a linewidth much smaller than the binding energy. In a 3D FL, the width of the QP $\left(\Gamma_{\mathrm{EDC}}\right)$, which is related to the MDC width $\left(\Gamma_{\mathrm{MDC}}\right)$, or the inverse of the QP scattering time $(\hbar / \tau)$, is a quadratic function of binding energy. To check whether $\mathrm{SrMnBi}_{2}$ can be described in such a framework, we analyzed the QP scattering rate by fitting the high-resolution MDCs along cut 1 and cut 2, as illustrated in Figs. 4(a) and 4(b), respectively.

The MDCs are fitted by using a constant flat background with single or double Lorentzian to reproduce the MDCs. The simple fitting method extracts the MDC linewidth very well. Except below $E_{B}=150 \mathrm{meV}$ along cut 1, where the band splits and two Lorentzian curves are necessary to reproduce 

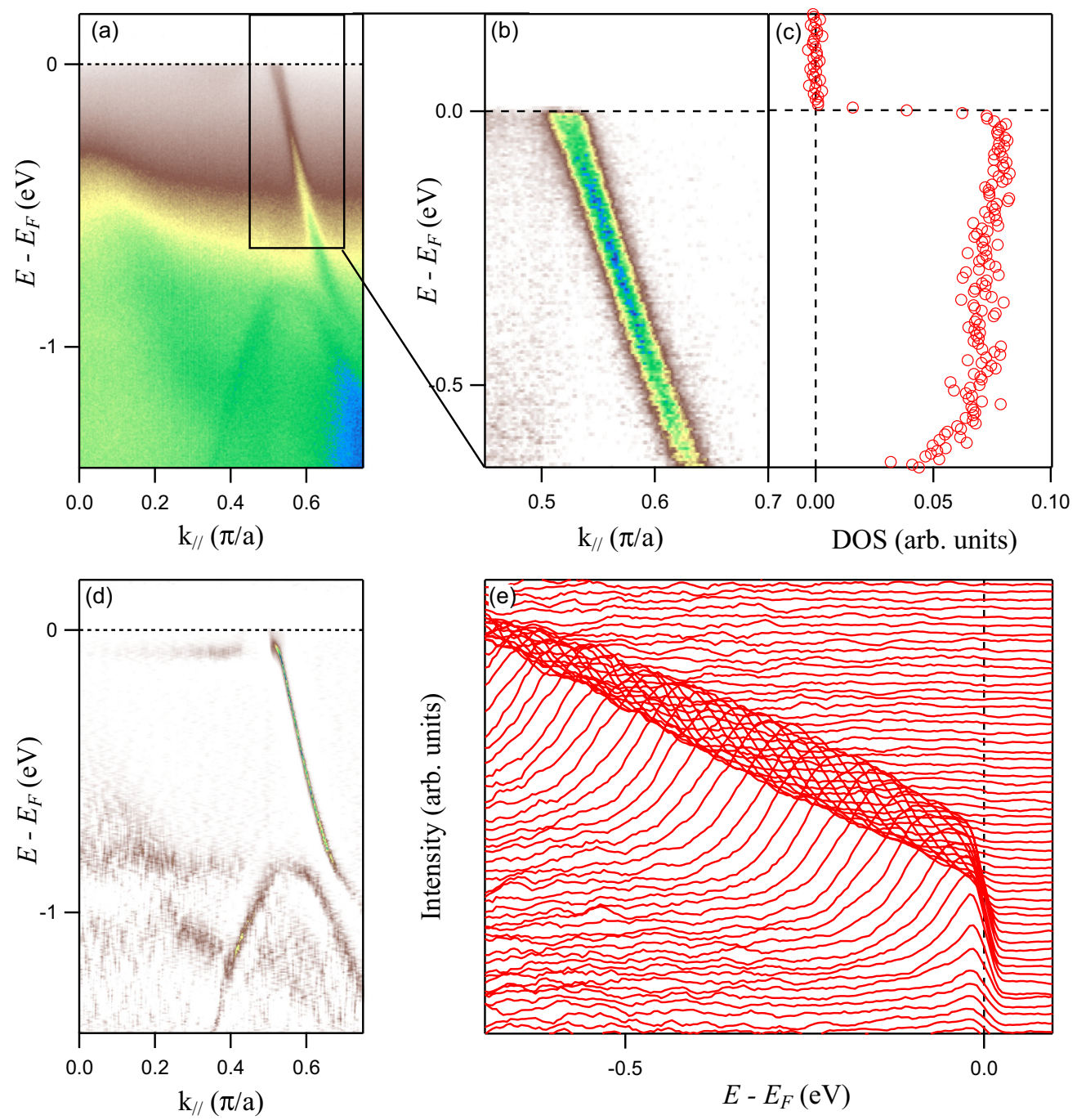

FIG. 3. (Color online) Constant line shape as a function of binding energy with high energy and momentum resolution at $T=10 \mathrm{~K}$. (a), (d) High-resolution intensity plot and the second derivative of the intensities along the cut 1 direction up to $1.4 \mathrm{eV}$ binding energy. (b), (e) Intensity plot and EDCs plot of the band dispersion with background removed. (c) The integrated intensity of (b) as a function of binding energy $\left[n_{\theta}(\omega)\right]$.

the results, a single Lorentzian curve is used to fit the MDCs up to 650 and $350 \mathrm{meV}$ of binding energy along cut 1 and cut 2 , respectively. We note that Lorentzian produce much better fits than Gaussian, suggesting that the spectral line shapes are at least not entirely resolution limited.

The values of the extracted QP MDC peak width as a function of binding energy along the two cuts are shown in

TABLE I. Density of states for parabolic dispersion and Diraccone-like dispersion at different dimensions.

\begin{tabular}{lcc}
\hline \hline & $\begin{array}{c}\text { Dirac dispersion } \\
\left(\omega=\omega_{0}+c k\right)\end{array}$ & $\begin{array}{c}\text { Parabolic dispersion } \\
\left(\omega=\omega_{0}+c k^{2}\right)\end{array}$ \\
\hline$\rho_{1 \mathrm{D}}(\omega)$ & $\frac{1}{c}$ & $\frac{1}{\sqrt{c\left(\omega-\omega_{0}\right)}}$ \\
\hline$\rho_{2 \mathrm{D}}(\omega)$ & $\frac{2 \pi}{c^{2}}\left(\omega-\omega_{0}\right)$ & $\frac{\pi}{c}$ \\
\hline$\rho_{3 \mathrm{D}}(\omega)$ & $\frac{4 \pi}{c^{3}}\left(\omega-\omega_{0}\right)^{2}$ & $\frac{2 \pi}{c} \sqrt{\left(\omega-\omega_{0}\right)}$ \\
\hline \hline
\end{tabular}

Figs. 4(c) and 4(d). The EDC peak widths $\left(\Gamma_{\text {EDC }}\right)$ are estimated by $\Gamma_{\mathrm{EDC}}=v_{F} \Gamma_{\mathrm{MDC}}$ and plotted in the corresponding figures. From the figures, in a wide energy range where the band dispersion is linear, the energy dependence of the MDC linewidth remains linearly, with different slope along cut 1 and cut 2, respectively. Considering the momentum resolution of the apparatus at the experimental setting, $\Delta k \sim 0.009 \AA$ at $h v=30 \mathrm{eV}$ photon energy, we remove its contribution from the fitted result using the formula $\Gamma_{0}=\sqrt{\Gamma_{\mathrm{MDC}}^{2}-\Delta_{k}^{2}}$, and plot the intrinsic peak width in Figs. 4(c) and 4(d). The intrinsic peak width $\Gamma_{0}$ also has a linear dependence with the binding energy. We estimate the QP lifetime $\tau \approx \hbar / \Gamma_{\mathrm{EDC}} \sim 33 \mathrm{fs}$ and the mean free path $l=v_{F} \tau \sim 43 \mathrm{~nm}$, comparable to the inelastic mean free path due to electron-electron interactions in graphene [18].

The linear dependence of the linewidth is not expected by the FL theory, where moderate electron-electron interactions are the dominant term. The linear dependence of the scattering rate in a wide range is inconsistent with the $3 \mathrm{D}$ FL theory $\left(1 / \tau \propto \omega^{2}\right)$ or the 2D FL theory $\left[1 / \tau \propto\left(\omega^{2} / \epsilon_{F}\right) \ln \left(4 \epsilon_{F} / \omega\right)\right]$. 

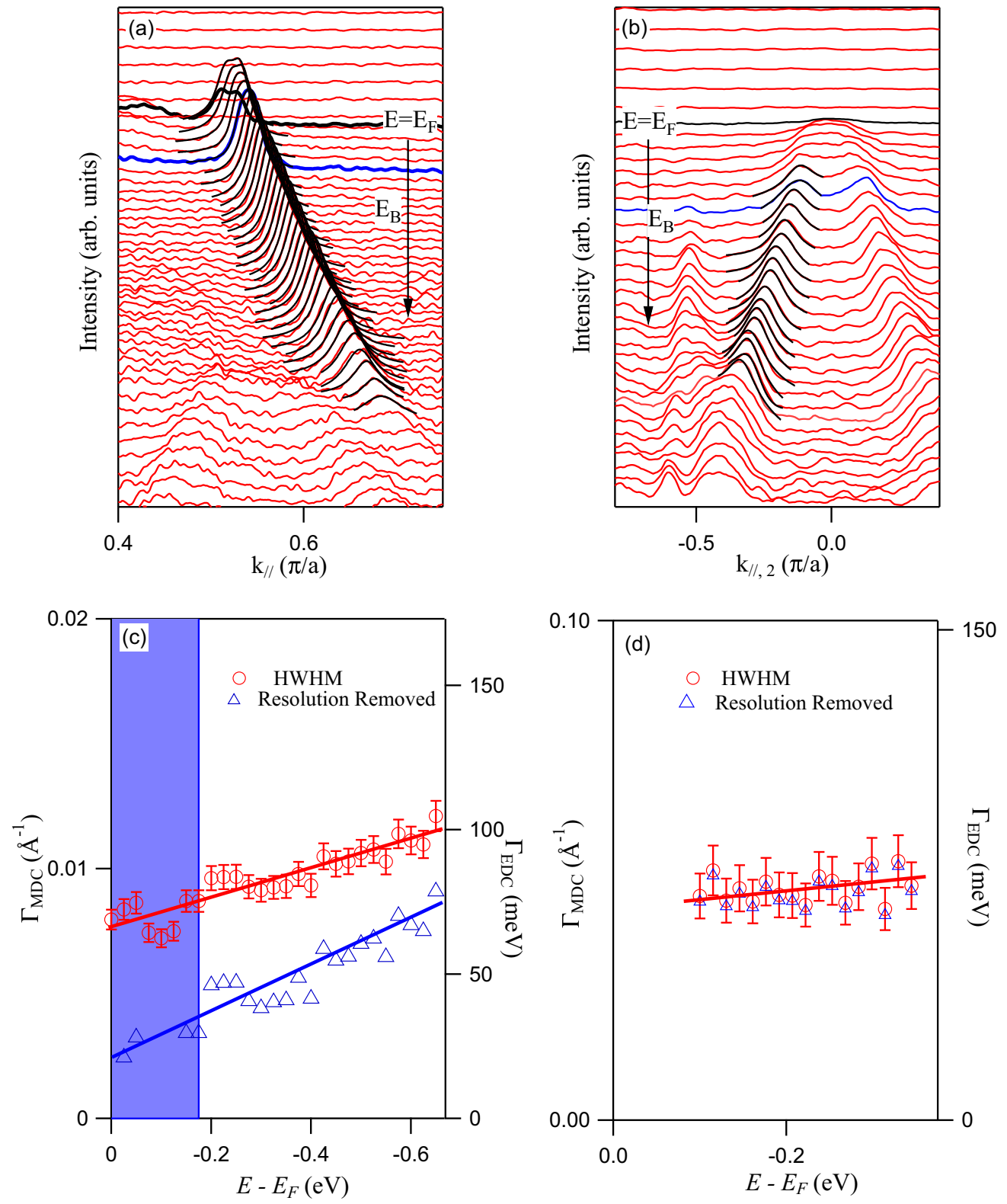

FIG. 4. (Color online) Unusual energy dependence of the quasiparticle width. (a) MDCs and Lorentz fitting of the high-resolution intensity along cut 1, as shown in Fig. 3(b). (b) MDCs and Lorentz-fitting results of intensity plot along the cut 2 direction. The black MDCs are recorded at $E_{F}$. The blue MDCs indicate the points between double-Lorentz and single-Lorentz fitting. (c), (d) Extracted half width of half maximum (HWHM) (red circles) in momentum (left axis) and energy (right axis, $\Gamma_{\mathrm{EDC}}=\Gamma_{\mathrm{MDC}} v_{F}$ ) of the Dirac fermions along cut 1 and cut 2 . The blue triangles show the intrinsic peak width, disposed of the momentum resolution by $\Gamma_{0}=\sqrt{\Gamma_{\mathrm{MDC}}^{2}-\Delta_{k}^{2}}$. The lines in (c) and (d) are a guide to the eye.

In contrast, this kind of unusual behavior has been observed in other 2D systems such as graphene and layered electron gas $[19,20]$, where the mechanism is not well understood yet. In graphene, the unusual linewidth was explained either by the decay channel due to the formation of a plasmon band in a layered electron gas, or by an electron-phonon coupling with linear dispersion, which contributes for only $1 / 5$ of the linewidth value experimentally observed [21], or by the strong electron-electron renormalization with similarity to the marginal Fermi-liquid (MFL) behavior [20]. It was also proposed that in pure graphene, the electron-electron interaction is a MFL whereas in doped graphene it is a regular 2D FL [22,23], but most measurements have actually been performed on unintentionally doped graphene [3]. With consideration of the possibilities in graphene, the linear increase of the linewidth in $\mathrm{SrMnBi}_{2}$ reflects the electronelectron interactions in a 2D system with a MFL behavior, as observed in undoped graphene.

Although $\mathrm{SrMnBi}_{2}$ is a $3 \mathrm{D}$ bulk crystal, our results suggest that the $\mathrm{SrBi}$ layer, which LDA calculations relate to the electronic states forming the Dirac cone, holds an unconventional 2D electron gas. Thus, the $\mathrm{SrMnBi}_{2}$ provides a clean system with undoped $\mathrm{SrBi}$ layers forming a 2D electron gas for the study of low-dimensional 
systems, and calls for further theoretical and experimental studies.

In summary, we observed highly anisotropic Dirac cones in bulk $\mathrm{SrMnBi}_{2}$. Well-defined quasiparticles with linear dispersion are observed over a wide energy range. Their linewidths (inverse of scattering rate) increase linearly with binding energy, and the integrated density of states is also linearly dependent on binding energy. These behaviors suggest the existence of a novel two-dimensional non-Fermi-liquid electron gas in this three-dimensional bulk material.
This work is supported by grants from National Science Foundation of China, National Basic Research Program of China (973 Program), Ministry of Education of China, Chinese Academy of Science, and SSSTC. The Synchrotron Radiation Center, WI, is supported by University of Wisconsin-Madison with supplemental support from facility users and the University of Wisconsin-Milwaukee. This work is based in part on research conducted at the Swiss Light Source, Paul Scherrer Institute, Villigen, Switzerland.
[1] M. A. Baranov, M. Yu. Kagan, and M. S. Mar'enko, Czech. J. Phys. 46, 2519 (1996).

[2] X. Zhou, C. Fang, W.-F. Tsai, and J. P. Hu, Phys. Rev. B 80, 245317 (2009).

[3] Aaron Bostwick, Taisuke Ohta, Thomas Seyller, Karsten Horn, and Eli Rotenberg, Nat. Phys. 3, 36 (2007).

[4] G. F. Giuliani and J. J. Quinn, Phys. Rev. B 26, 4421 (1982).

[5] H. Çelik, M. Cankurtaran, A. Bayrakli, E. Tiras, and N. Balkan, Semicond. Sci. Technol. 12, 389 (1997).

[6] Yuanbo Zhang, Yan-Wen Tan, Horst L. Stormer, and Philip Kim, Nature (London) 438, 201 (2005).

[7] B. Andrei Bernevig, Taylor L. Hughes, and Shou-Cheng Zhang, Science 314, 1757 (2006).

[8] K. v. Klitzing, G. Dorda, and M. Pepper, Phys. Rev. Lett. 45, 494 (1980).

[9] Tsuneya Ando, Alan B. Fowler, and Frank Stern, Rev. Mod. Phys. 54, 437 (1982).

[10] Kefeng Wang, Limin Wang, and C. Petrovic, Appl. Phys. Lett. 100, 112111 (2012).

[11] J. Park, G. Lee, F. Wolff-Fabris, Y. Y. Koh, M. J. Eom, Y. K. Kim, M. A. Farhan, Y. J. Jo, C. Kim, J. H. Shim, and J. S. Kim, Phys. Rev. Lett. 107, 126402 (2011).

[12] K. Wang, D. Graf, L. Wang, H. Lei, S. W. Tozer, and C. Petrovic, Phys. Rev. B 85, 041101 (2012).
[13] J. B. He, D. M. Wang, and G. F. Chen, Appl. Phys. Lett. 100, 112405 (2012).

[14] J. K. Wang, L. L. Zhao, Q. Yin, G. Kotliar, M. S. Kim, M. C. Aronson, and E. Morosan, Phys. Rev. B 84, 064428 (2011).

[15] Cheol-Hwan Park, Li Yang, Young-Woo Son, Marvin L. Cohen, and Steven G. Louie, Nat. Phys. 4, 213 (2008).

[16] Christian R. Ast, Gero Wittich, Peter Wahl, Ralf Vogelgesang, Daniela Pacilé, Mihaela C. Falub, Luca Moreschini, Marco Papagno, Marco Grioni, and Klaus Kern, Phys. Rev. B 75, 201401 (2007).

[17] D. S. Saraga and D. Loss, Phys. Rev. B 72, 195319 (2005).

[18] Choongyu Hwang, David A. Siegel, Sung-Kwan Mo, William Regan, Ariel Ismach, Yuegang Zhang, Alex Zettl, and Alessandra Lanzara, Sci. Rep. 2, 590 (2012).

[19] David A. Siegel, Cheol-Hwan Park, Choongyu Hwang, Jack Deslippe, Alexei V. Fedorov, Steven G. Louie, and Alessandra Lanzara, Proc. Natl. Acad. Sci. USA 108, 11365 (2011).

[20] S. Xu, J. Cao, C. C. Miller, D. A. Mantell, R. J. D. Miller, and Y. Gao, Phys. Rev. Lett. 76, 483 (1996).

[21] M. Calandra and F. Mauri, Phys. Rev. B 76, 205411 (2007).

[22] S. Das Sarma, E. H. Hwang, and W.-K. Tse, Phys. Rev. B 75, 121406 (2007).

[23] C.-H. Park, F. Giustino, M. L. Cohen, and S. G. Louie, Phys. Rev. Lett. 99, 086804 (2007). 